\title{
MOTION CONTROL OF A HYDRAULIC STEWART PLATFORM WITH COMPUTED FORCE FEEDBACK
}

\author{
Chih-Chin Hsu and I-Kong Fong* \\ Department of Electrical Engineering \\ National Taiwan University \\ Taipei, Taiwan 106, R.O.C.
}

Key Words: Stewart platform, force feedback control, gyroscope.

\begin{abstract}
On a Stewart platform with six hydraulic links, we mount three gyroscopes to measure the angular motion rates of its upper platform. Using these rate signals and the link lengths measured by LVDTs, we compute the orientation and position of the upper platform, as well as the static force acting on each link. These forces are put into a dynamic model of the hydraulic links based on which we determine the feedback gains for controlling the link lengths. Experiments on the platform with an unbalanced payload show that utilizing gains determined this way generates better results than utilizing a constant gain for all links.
\end{abstract}

\section{INTRODUCTION}

A Stewart platform is a parallel manipulator with a movable upper platform and a fixed base connected by six variable length links. When the length of each link is controlled properly, the upper platform is capable of performing six degree-of-freedom motion within its working space. With high stiffness, simple inverse kinematics and good dynamic response characteristics, the Stewart platform has been the subject of many studies including kinematics and dynamics analysis (Cheok et al., 1993; Do and Yang, 1988), practical design considerations (Fichter, 1986), working space and singularities determination (Liu et al., 1993), controller synthesis (Charles et al., 1993; Kosuge et al., 1996; Wen et al., 1998a, 1998b), and many application developments (Grant and Reid, 1997; Salcudean et al., 1994). Figure 1 is a picture of the hydraulic Stewart platform assembled in the Advanced Control Laboratory of the Department of Electrical Engineering, National Taiwan University.

In this paper we investigate the motion control problem of the hydraulic Stewart platform shown in Fig. 1. A similar problem is studied in (Charles et al., 1993) for a much smaller platform, with links driven by DC servo motors, which has a different set of dynamic characteristics from the platform considered here. The work of Kosuge et al. (1996) involves a hydraulic Stewart platform, but the control purpose is mainly force exertion. With the parallel structure of the Stewart platform, it is easy to comprehend, that, when one tries to control its link lengths, one has to deal with interactions among all links. We see in our dynamic model of the hydraulic links that such interactions are primarily through forces acting on the links. In (Wen et al., 1998a, 1998b) we treat these interactions as uncertain parameters of the dynamic model, and successfully find robust controllers for handling trajectory commands that are known in advance. Here we adopt a more explicit approach and seek to decide the interactions for the controller to cancel, at least partially. We mount three gyroscopes to measure the angular motion rates of the upper platform, which together with the link lengths

*Correspondence addressee 


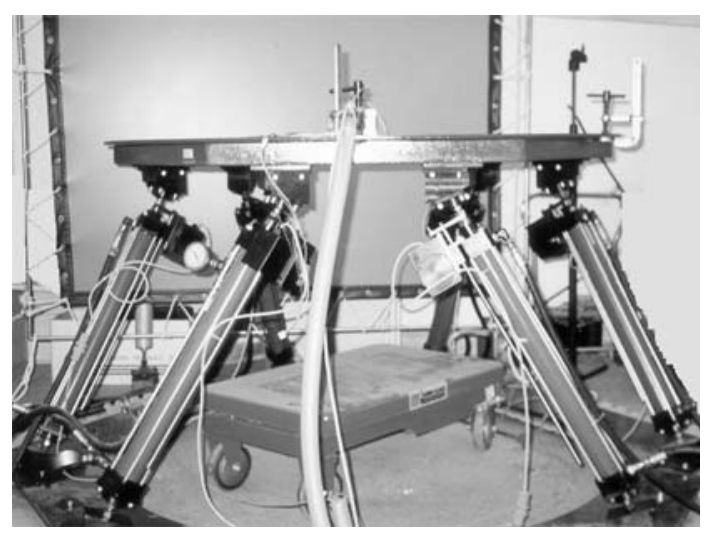

Fig. 1 A hydraulic Stewart platform

measured by LVDTs enable us to compute the orientation and position of the upper platform, as well as the static force acting on each link. These static forces are only approximation of the true interactions, which are dynamic forces. However, due to the fact that the hydraulic platform is not a fast moving apparatus, and according to our experience in (Wen et al., 1998a, 1998b), we believe that such an approximation is sufficiently good. Results from experiments performed in this work also support this usage, which deliberately worsen the interactions by putting a heavy unbalanced payload on the platform. It is worth noting that our use of three gyroscopes along with six LVDTs offers an alternative way of solving the forward kinematics problem to that proposed in (Cheok et al., 1993), where a total of nine LVDTs are wisely used to find the position of the upper platform. Our method lets us compute both position and orientation of the upper platform quickly enough for the purpose of realtime control.

The rest of this paper is organized as follows. In Section II, we derive the kinematics and static force equations, which will be used to determine the position of the upper platform and the static forces acting on the links. The orientation of the upper platform is obtained in Section III, where a system of three rate gyroscopes, set to serve our purpose, is described. In Section IV, we introduce the dynamic model for a single link with its hydraulic actuator. In addition, we propose a controller with an independently adjustable gain for each link based on the force the link withstands. Experimental results are given in Section $\mathrm{V}$ to compare the performance of the proposed controller with that of a constant gain controller. Finally, conclusions are given in Section VI.

\section{KINEMATICS AND STATIC FORCE EQUATIONS}

Before we start, some remarks regarding the notational convention adopted in this paper. We use boldface upper and lower cases letters to denote matrix and vector variables, respectively. In addition, we have three dimensional Cartesian space vectors like $\overrightarrow{A B}$, which is the vector from point $A$ to point $B$. Also, in three dimensional Cartesian space, we may have different coordinate frames. For example, frame $\{F\}$ is the one that has the origin $O_{F}$ and rectangular coordinate $X Y Z_{F}$ with the right-hand perpendicular axes $X_{F}, Y_{F}, Z_{F}$ and corresponding unit vectors $\boldsymbol{i}_{F}, \boldsymbol{j}_{F}$, $\boldsymbol{k}_{F}$. For vectors $\boldsymbol{v}$ and $\overrightarrow{A B}, \boldsymbol{v}^{F}$ and $\overrightarrow{A B}$ denote their $3 \times 1$ representation column vectors with respect to $\boldsymbol{i}_{F}$, $\boldsymbol{j}_{F}, \boldsymbol{k}_{F}$. However, the superscript $T$ is reserved for the transpose operation of matrices and column/row vectors. Finally, $\|\cdot\|_{2}$ stands for the Euclidean norm of a vector. In particular, it is equal to the length of its argument vector in three dimensional Cartesian space.

\section{Kinematic Equations}

The basic geometry of our Stewart platform and associated kinematic equations are described in this subsection. Moreover, we shall show how to determine the position of the upper platform quickly if its orientation and all link lengths are known.

Figure 2(a) is a simplified sketch of the Stewart platform to be discussed, where we attach frames $\{P\}$ and $\{B\}$ to the upper platform and the lower base, respectively. We also attach frame $\left\{J_{i}\right\}$ to the $i$ th link, which is connected to the platform and the base through two universal ball joints at points $P_{i}$ and $B_{i}$, respectively. Marked in Fig. 2(b), which is a top view, these joints lie on circles centered on centroids $O_{P}$ and $O_{B}$ with radius $R_{P}$ and $R_{B}$, respectively. The angle between $P_{1}$ and $P_{2}$ is $\theta_{P}$, and the angle between $B_{1}$ and $B_{2}$ is $\theta_{B}$. Other points distribute, symmetrically, along the circles with a $\frac{2}{3} \pi$ span. The $X_{B}$ axis of frame $\{B\}$ is perpendicular to the line connecting the two joints $B_{1}$ and $B_{6}$, and the $X_{B}-Y_{B}$ plane coincides with the base plane. The $Z_{B}$ axis is determined by the right hand rule. Fixed to the upper platform, the reference coordinate $X Y Z_{P}$ of frame $\{P\}$ is similarly defined. As to the $i$ th link frame $\left\{J_{i}\right\}, i=1, \ldots, 6$, the origin $O_{J_{i}}$ is at the point $B_{i}$, the $X_{J_{i}}$-axis points toward $P_{i}$, the $Y_{J_{i}}$ axis is parallel to the cross product of $\vec{B}_{i} P_{i}$ and $-\boldsymbol{k}_{B}$, and the $Z_{J_{i}}$ axis is determined by the right hand rule. For the sake of simplicity, hereafter we write $O_{J_{i}}$ as $O_{i}, X_{J_{i}}$ as $X_{i}$, and $i_{J_{i}}$ as $\boldsymbol{i}_{i}$, etc.

Using the notations defined above, we consider the relative position and orientation of frame $\{P\}$ with respect to frame $\{B\}$ first. The relative position can be described by ${\overrightarrow{O_{B} O_{P}}}^{B}=\left[\begin{array}{lll}x & y & z\end{array}\right]^{T}$, while the orientation can be described by three Euler angles $\alpha, \beta$, and $\gamma$, obtained by performing the following sequence of rotations to make the inertial frame $\{B\}$ become frame 


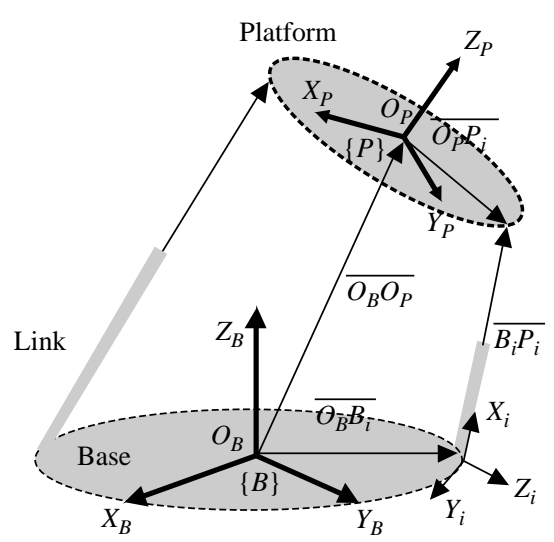

(a)

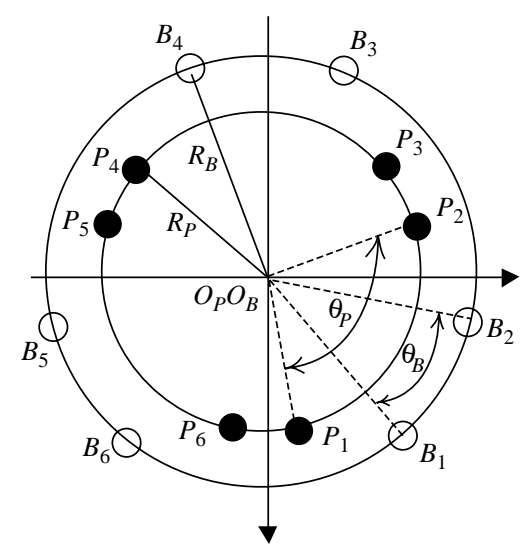

(b)

Fig. 2 (a) Basic geometry of the Stewart platform and the attached frames. (b) Top view of the ball joints arrangement on the base and upper platform.

$\{P\}$ : first rotate frame $\{B\}$ about the $Z_{B}$-axis a (yaw) angle $\alpha$ to get a frame $\left\{B^{\prime}\right\}$, then rotate $\left\{B^{\prime}\right\}$ about its $Y_{B^{\prime}}$-axis a (pitch) angle $\beta$ to get another frame $\left\{B^{\prime \prime}\right\}$, and finally rotate $\left\{B^{\prime \prime}\right\}$ about its $X_{B^{\prime \prime}}$-axis a (roll) angle $\gamma$ to obtain the frame $\{P\}$. This orientation representation also gives an Euler orientation matrix $\boldsymbol{R}_{P}^{B}$ equal to

$$
\begin{aligned}
& \left.\begin{array}{lr}
-\sin (\beta) & \cos (\beta) \sin (\gamma) \\
\cos (\alpha) \sin (\beta) \cos (\gamma)+\sin (\alpha) \sin (\gamma) \\
\sin (\alpha) \sin (\beta) \cos (\gamma)-\cos (\alpha) \sin (\gamma) \\
\cos (\beta) \cos (\gamma)
\end{array}\right] \\
& \equiv\left[\begin{array}{lll}
r_{11} & r_{12} & r_{13} \\
r_{21} & r_{22} & r_{23} \\
r_{31} & r_{32} & r_{33}
\end{array}\right],
\end{aligned}
$$$$
[\cos (\alpha) \cos (\beta) \cos (\alpha) \sin (\beta) \sin (\gamma)-\sin (\alpha) \cos (\gamma)
$$$$
\sin (\alpha) \cos (\beta) \quad \sin (\alpha) \sin (\beta) \sin (\gamma)+\cos (\alpha) \cos (\gamma)
$$

which also serves as the coordinate transformation matrix from the frame $\{P\}$ to the frame $\{B\}$. Now if we denote the angle between $\vec{O}_{B} B_{i}$ and the $X_{B}$-axis by $\Lambda_{i}$, and the angle between ${\overrightarrow{O_{P} P}}_{i}$ and the $X_{P^{-}}$axis by $\lambda_{i}$, $i=1,2, \ldots, 6$, then we have

$$
\begin{array}{ll}
\Lambda_{i}=\frac{1}{3} \pi \cdot i-\frac{\theta_{B}}{2}, & i=1,3,5, \\
\lambda_{i}=\frac{1}{3} \pi \cdot i-\frac{\theta_{P}}{2}, & i=1,3,5, \\
\Lambda_{i}=\Lambda_{i-1}+\theta_{B}, & i=2,4,6, \\
\lambda_{i}=\lambda_{i-1}+\theta_{P}, & i=2,4,6 .
\end{array}
$$

Thus

$$
\begin{aligned}
{\overrightarrow{O_{P} P_{i}}}^{P} & =\left[\begin{array}{lll}
R_{P} \cos \left(\lambda_{i}\right) & R_{P} \sin \left(\lambda_{i}\right) & 0
\end{array}\right]^{T} \\
& \equiv\left[\begin{array}{lll}
P_{i x} & P_{i y} & 0
\end{array}\right]^{T}, \quad i=1,2, \ldots, 6, \\
{\overrightarrow{O_{B} B_{i}}}^{B} & =\left[\begin{array}{lll}
R_{B} \cos \left(\Lambda_{i}\right) & R_{B} \sin \left(\Lambda_{i}\right) & 0
\end{array}\right]^{T} \\
& \equiv\left[\begin{array}{lll}
B_{i x} & B_{i y} & 0
\end{array}\right]^{T}, \quad i=1,2, \ldots, 6 .
\end{aligned}
$$

With these preparations, it is easy to see from Fig. 2(a) that the link vector $\vec{B}_{i} P_{i}$ expressed with respect to the frame $\{B\}$ is the sum of three vectors

$$
{\overrightarrow{B_{i} P}}_{i}^{B}={\overrightarrow{O_{B} O_{P}}}^{B}+\boldsymbol{R}_{P}^{B} \cdot{\overrightarrow{O_{P} P}}_{i}^{P}-{\overrightarrow{O_{B} B_{i}}}^{B} .
$$

Hence the length $l_{i}$ of the $i$ th link can be computed from

$$
l_{i}=\left\|{\overrightarrow{B_{i} P_{i}}}^{B}\right\|_{2} .
$$

Eq. (6) represents the closed-form solution to the inverse kinematics problem. More explicitly, it can be written as

$$
\begin{aligned}
& l_{i}^{2}=x^{2}+y^{2}+z^{2}+R_{P}^{2}+R_{B}^{2} \\
& +2\left(r_{11} P_{i x}+r_{12} P_{i y}\right)\left(x-B_{i x}\right) \\
& +2\left(r_{21} P_{i x}+r_{22} P_{i y}\right)\left(y-B_{i y}\right) \\
& +2\left(r_{31} P_{i x}+r_{32} P_{i y}\right) z \\
& -2\left(B_{i x} x+B_{i y} y\right) .
\end{aligned}
$$

Subtracting (7) with a general index $i$ from the same equation with the index $i$ set to 1 leads to 


$$
\begin{aligned}
& {\left[r_{11}\left(P_{1 x}-P_{i x}\right)+r_{12}\left(P_{1 y}-P_{i y}\right)-\left(B_{1 x}-B_{i x}\right)\right] x} \\
& +\left[r_{21}\left(P_{1 x}-P_{i x}\right)+r_{22}\left(P_{1 y}-P_{i y}\right)-\left(B_{1 y}-B_{i y}\right)\right] y \\
& +\left[r_{31}\left(P_{1 x}-P_{i x}\right)+r_{32}\left(P_{1 y}-P_{i y}\right)\right] z \\
& =\left[\left(r_{11} P_{1 x}+r_{12} P_{1 y}\right) B_{1 x}-\left(r_{11} P_{i x}+r_{12} P_{i y}\right) B_{i x}\right. \\
& \left.+\left(r_{21} P_{1 x}+r_{22} P_{1 y}\right) B_{1 y}-\left(r_{21} P_{i x}+r_{22} P_{i y}\right) B_{i y}\right] \\
& +\frac{1}{2}\left(l_{1}^{2}-l_{i}^{2}\right) .
\end{aligned}
$$

From (8) with $i=2,3, \ldots, 6$, we have a system of five linear equations about the variables $x, y$, and $z$, which can be put into the matrix form

$$
\boldsymbol{M} \cdot \boldsymbol{N}\left[\begin{array}{l}
x \\
y \\
z
\end{array}\right]=\boldsymbol{b} \equiv\left[\begin{array}{c}
b_{1} \\
b_{2} \\
\vdots \\
b_{5}
\end{array}\right]
$$

with

$$
M=\left[\begin{array}{llll}
\left(P_{1 x}-P_{2 x}\right) & \left(P_{1 y}-P_{2 y}\right) & \left(B_{2 x}-B_{1 x}\right) & \left(B_{2 y}-B_{1 y}\right) \\
\left(P_{1 x}-P_{3 x}\right) & \left(P_{1 y}-P_{3 y}\right) & \left(B_{3 x}-B_{1 x}\right) & \left(B_{3 y}-B_{1 y}\right) \\
\left(P_{1 x}-P_{4 x}\right) & \left(P_{1 y}-P_{4 y}\right) & \left(B_{4 x}-B_{1 x}\right) & \left(B_{4 y}-B_{1 y}\right) \\
\left(P_{1 x}-P_{5 x}\right) & \left(P_{1 y}-P_{5 y}\right) & \left(B_{5 x}-B_{1 x}\right) & \left(B_{5 y}-B_{1 y}\right) \\
\left(P_{1 x}-P_{6 x}\right) & \left(P_{1 y}-P_{6 y}\right) & \left(B_{6 x}-B_{1 x}\right) & \left(B_{6 y}-B_{1 y}\right)
\end{array}\right]
$$

$$
\begin{aligned}
N= & {\left[\begin{array}{ccc}
r_{11} & r_{21} & r_{31} \\
r_{12} & r_{22} & r_{32} \\
1 & 0 & 0 \\
0 & 1 & 0
\end{array}\right] } \\
b_{j}= & \frac{1}{2}\left(l_{1}^{2}-l_{j+1}^{2}\right)+\left(r_{11} P_{1 x}+r_{12} P_{1 y}\right) B_{1 x} \\
& -\left[r_{11} P_{(j+1) x}+r_{12} P_{(j+1) y}\right] B_{(j+1) x} \\
& +\left(r_{21} P_{1 x}+r_{22} P_{1 y}\right) B_{1 y} \\
& -\left[r_{21} P_{(j+1) x}+r_{22} P_{(j+1) y}\right] B_{(j+1) y}, \\
& j=1,2, \ldots, 5 .
\end{aligned}
$$

Note that $\boldsymbol{M}$ is a constant matrix decided by the geometric parameters $R_{P}, R_{B}, \theta_{P}$, and $\theta_{B}$. In our case it has full column rank. The matrix $N$ also has full column rank unless $r_{31}$ and $r_{32}$ become zeros at the same time, but since we shall only use (9) to find $x$ and $y$, the first two rows of $N$ will play no roles. As to the vector $\boldsymbol{b}$, besides the platform geometric parameters, we must know the orientation angles of the upper platform and the link lengths. For the latter we have six LVDTs mounted on the links, and for the former we use the method to be described in Section III. Given all the needed information, we can multiply the pseudo-inverse of $\boldsymbol{M}$ to both sides of (9) to form

$$
\boldsymbol{N}\left[\begin{array}{l}
x \\
y \\
z
\end{array}\right]=\left(\boldsymbol{M}^{T} \boldsymbol{M}\right)^{-1} \boldsymbol{M}^{T} \boldsymbol{b} \equiv \tilde{\boldsymbol{b}}=\left[\begin{array}{c}
\tilde{b}_{1} \\
\tilde{b}_{2} \\
\tilde{b}_{3} \\
\tilde{b}_{4}
\end{array}\right]
$$

Due to the simple structure of the lower half of the matrix $N$, it is easy to conclude that

$$
\begin{aligned}
& x=\tilde{b}_{3}, \\
& y=\tilde{b}_{4} .
\end{aligned}
$$

Note that to accelerate the computation, we only need to multiply the last two rows of $\left(\boldsymbol{M}^{T} \boldsymbol{M}\right)^{-1} \boldsymbol{M}^{T}$ to $\boldsymbol{b}$, since $\tilde{b}_{1}$ and $\tilde{b}_{2}$ are useless to us. With $x$ and $y$ in hand, we turn back to (7) for determining $z$. Adding (7) with $i=1,2, \ldots, 6$ together, dividing the sum by 6 , and using some identities of trigonometric functions, we get

$$
\begin{aligned}
z= & \left\{\frac{1}{6} \sum_{i=1}^{6} l_{i}^{2}+\frac{1}{3} \sum_{i=1}^{6}\left[\left(r_{11} P_{i x}+r_{12} P_{i y}\right) B_{i x}\right.\right. \\
& \left.\left.+\left(r_{21} P_{i x}+r_{22} P_{i y}\right) B_{i y}\right]-x^{2}-y^{2}-R_{P}^{2}-R_{B}^{2}\right\}^{1 / 2} .
\end{aligned}
$$

Thus, we solve "half" of the complicated forward kinematics problem by assuming that in addition to the link lengths, we know the orientation of the upper platform. Moreover, the required computation time to get the unique solution is short enough that it can be utilized in our real-time feedback system.

\section{Static Force Equations}

The $X_{i}$-axis of the $i$ th link coincides with ${\overrightarrow{B_{i}}}_{i}$, so the unit vector $\boldsymbol{i}_{i}$ can be expanded as

$$
\boldsymbol{i}_{i}=\frac{{\overrightarrow{B_{i} P}}_{i}}{\|{\overrightarrow{B_{i} P_{i}} \|_{2}}_{x_{x}}} \equiv i_{i_{x}} \boldsymbol{i}_{B}+i_{i_{y}} \boldsymbol{j}_{B}+i_{i_{z}} \boldsymbol{k}_{B},
$$

where $i_{i_{x}}, i_{i_{y}}$, and $i_{i_{z}}$ are exactly the three components of $\vec{B}_{i}{ }_{i}{ }^{B}$ in (5) divided by $i_{i}$. Furthermore, from the definition of the frame $\left\{J_{i}\right\}$ we have 


$$
\begin{aligned}
& \boldsymbol{j}_{i}=\frac{\boldsymbol{i}_{i} \times\left(-\boldsymbol{k}_{B}\right)}{\left\|\boldsymbol{i}_{i} \times\left(-\boldsymbol{k}_{B}\right)\right\|_{2}} \equiv j_{i_{x}} \boldsymbol{i}_{B}+j_{i_{y}} \boldsymbol{j}_{B}+j_{i_{z}} \boldsymbol{k}_{B}, \\
& \boldsymbol{k}_{i}=\boldsymbol{i}_{i} \times \boldsymbol{j}_{i} \equiv k_{i_{x}} \boldsymbol{i}_{B}+k_{i_{y}} \boldsymbol{j}_{B}+k_{i_{z}} \boldsymbol{k}_{B} .
\end{aligned}
$$

Note that by the rule of cross product, $j_{i_{z}}=0$ for $i=1$, $2, \ldots, 6$, in (18). With these quantities, the coordinate transformation matrix from frame $\{B\}$ to frame $\left\{J_{i}\right\}$ can be constructed as

$$
\boldsymbol{R}_{B}^{J_{i}}=\left[\begin{array}{lll}
\boldsymbol{i}_{i}^{B} & \boldsymbol{j}_{i}^{B} & \boldsymbol{k}_{i}^{B}
\end{array}\right]^{T} \equiv\left[\begin{array}{ccc}
i_{i_{x}} & i_{i_{y}} & i_{i_{z}} \\
j_{i_{x}} & j_{i_{y}} & j_{i_{z}} \\
k_{i_{x}} & k_{i_{y}} & k_{i_{z}}
\end{array}\right]
$$

Hence, the coordinate transformation matrix $\boldsymbol{T}_{i}^{P}$ from the frame $\left\{J_{i}\right\}$ to the frame $\{P\}$ can be constructed by combining two transformations as

$$
\boldsymbol{T}_{i}^{P}=\boldsymbol{R}_{B}^{P} \cdot \boldsymbol{R}_{J_{i}}^{B}=\boldsymbol{R}_{P}^{B^{T}} \cdot \boldsymbol{R}_{B}^{J_{i}^{T}} \equiv\left[\begin{array}{ccc}
t_{i_{11}} & t_{i_{12}} & t_{i_{13}} \\
t_{i_{21}} & t_{i_{22}} & t_{i_{23}} \\
t_{i_{31}} & t_{i_{32}} & t_{i_{33}}
\end{array}\right]
$$

Now we derive the equations for the static forces acting on the links. Because we are concerned with the forces that the hydraulic actuators must generate, we shall focus on the forces along the direction of $\boldsymbol{i}_{i}$. Suppose the Stewart platform is a rigid body in a balanced and still status, with its base fixed to the earth. Then the net forces and moments acting on the upper platform and all links are zeros. Let us consider the free-body diagrams of the $i$ th link and the upper platform separately. Assume the force that the upper platform exerts on the $i$ th link is $f_{P_{i}}$, and the weights of the upper platform and the $i$ th link are $w_{P}$ and $w_{i}$, respectively. Additionally, the center of mass of the upper platform is assumed to be its center position, and the center of the mass of each link is assumed to be its middle position. In our practical situation, the link is a hydraulic cylinder, so its center of mass deviates from the middle position a little when its length changes, but this detail can be ignored because the resultant error is small. As to the upper platform, when there is only symmetric payload, the assumption about the position of its center of mass is valid, but, when there is unbalanced payload, we have to estimate the position of the mass center, and adjust the term ${\overrightarrow{O_{P} P}}_{i}$ in (28) below, accordingly. Fortunately, the system of equations derived this way has similar form to that obtained in the following derivation.

(i) The net moment due to the weight vector $\boldsymbol{w}_{i}=$ $-w_{i} \cdot \boldsymbol{k}_{B}$ of the $i$ th link and the force $\boldsymbol{f}_{P_{i}}$ acting on the $i$ th link about point $B_{i}$ is zero. Thus

$$
\begin{aligned}
& 2 \boldsymbol{r}_{i} \times \boldsymbol{f}_{P_{i}}+\boldsymbol{r}_{i} \times \boldsymbol{w}_{i} \\
& =2 \boldsymbol{r}_{i} \times \boldsymbol{f}_{P_{i}}+\boldsymbol{r}_{i} \times\left(w_{i_{x}} \boldsymbol{i}_{i}+w_{i_{y}} \boldsymbol{j}_{i}+w_{i_{z}} \boldsymbol{k}_{i}\right)=\mathbf{0},
\end{aligned}
$$

where

$$
\begin{aligned}
& \boldsymbol{f}_{P_{i}}=f_{P_{i x}} \boldsymbol{i}_{i}+f_{P_{i y}} \boldsymbol{j}_{i}+f_{P_{i z}} \boldsymbol{k}_{i}, \\
& \boldsymbol{r}_{i}=\frac{1}{2} l_{i} \boldsymbol{i}_{i}, \\
& {\left[\begin{array}{l}
w_{i_{x}} \\
w_{i_{y}} \\
w_{i_{z}}
\end{array}\right]=\boldsymbol{R}_{B}^{J_{i}} \cdot\left[\begin{array}{c}
0 \\
0 \\
-w_{i}
\end{array}\right] .}
\end{aligned}
$$

Solving (22), we get

$$
\begin{aligned}
& f_{P_{i y}}=\frac{1}{2} j_{i_{z}} w_{i}=0, \\
& f_{P_{i z}}=\frac{1}{2} k_{i_{z}} w_{i} .
\end{aligned}
$$

(ii) The net moment acting on the upper platform about its center of mass is zero. Thus

$$
\sum_{i=1}^{6}{\overrightarrow{O_{P} P}}_{i} \times\left(-f_{i_{x}} \boldsymbol{i}_{P}-f_{i_{y}} \boldsymbol{j}_{P}-f_{i_{z}} \boldsymbol{k}_{P}\right)=0
$$

where

$$
\left[\begin{array}{l}
f_{i_{x}} \\
f_{i_{y}} \\
f_{i_{z}}
\end{array}\right]=\boldsymbol{T}_{i}^{P} \cdot\left[\begin{array}{l}
f_{P_{i x}} \\
f_{P_{i y}} \\
f_{P_{i z}}
\end{array}\right]=\boldsymbol{T}_{i}^{P} \cdot\left[\begin{array}{c}
f_{P_{i x}} \\
0 \\
k_{i_{z}} w_{i} / 2
\end{array}\right]
$$

(iii) The net force due to the weight of the platform $-w_{P} \boldsymbol{k}_{B}$ and the forces $\boldsymbol{f}_{P_{i}}, i=1,2, \ldots, 6$, acting on the upper platform is zero. Thus

$$
\left[\begin{array}{c}
0 \\
0 \\
-w_{P}
\end{array}\right]-\sum_{i=1}^{6} \boldsymbol{R}_{J_{i}}^{B} \cdot\left[\begin{array}{c}
f_{P_{i x}} \\
0 \\
k_{i_{z}} w_{i} / 2
\end{array}\right]=0
$$

Rearranging (28) and (30), we have six equations in a compact matrix form

$$
\boldsymbol{D} \cdot \boldsymbol{f}_{p}=\boldsymbol{p}
$$

where the variable vector $f_{P}=\left[f_{P_{1_{x}}} f_{P_{2 x}} \ldots f_{P_{6_{x}}}\right]^{T}$, the coefficient vector 


$$
\boldsymbol{p}=\frac{1}{2} \sum_{i=1}^{6}\left[\begin{array}{c}
\left(t_{i_{23}} P_{i x} k_{i_{z}} w_{i}-t_{i_{13}} P_{i y} k_{i_{z}} w_{i}\right) \\
-t_{i_{33}} P_{i x} k_{i_{z}} w_{i} \\
t_{i_{33} P_{i y} k_{i_{z}} w_{i}} \\
k_{i_{x}} k_{i_{z}} w_{i} \\
k_{i_{y} k_{i z} w_{i}} w_{i} \\
k_{i_{z}}^{2} w_{i}
\end{array}\right]+\left[\begin{array}{c}
0 \\
0 \\
0 \\
0 \\
0 \\
w_{P}
\end{array}\right],
$$

the coefficient matrix $D=\left[\begin{array}{llll}d_{1} & d_{2} & \ldots & d_{6}\end{array}\right]$, and

$$
\boldsymbol{d}_{i}=\left[\begin{array}{c}
\left(-P_{i x} t_{i_{21}}+P_{i y} t_{i_{11}}\right) \\
P_{i x} t_{i_{31}} \\
-P_{i y} t_{i_{31}} \\
-i_{i_{x}} \\
-i_{i_{y}} \\
-i_{i_{z}}
\end{array}\right]
$$

The system of equations (31) can be uniquely solved provided $\boldsymbol{D}$ is nonsingular. Note that $\boldsymbol{D}$ plays the same role as a force transformation matrix in (Bhaskar and Mruthyunjaya, 1998) and (Bhaskar and Mruthyunjaya, 2000), which transforms three forces and three moments of end-effector into linear actuator forces of each link. When $\boldsymbol{D}$ is singular, the transformation degenerates and it means that some loads on the platform can not be supported by the actuator forces $\boldsymbol{f}_{p}$. Note, also, that the inverse velocity kinematics are essentially described by $\boldsymbol{D}^{T}$ (Tsai, 1999), which connects the velocity of each link to the rates of position and orientation of the upper platform. The singularity condition of $\boldsymbol{D}$ is characterized by zeroing its determinant, but finding the closed form of the roots is very difficult, and regarded as an open problem in (Bhaskar and Mruthyunjaya, 2000). In order to avoid the singularity condition, we take two measures. The first one is carried out when a trajectory command is given in advance. We can, off-line, examine the condition numbers of $\boldsymbol{D}$ to decide whether the platform will go through a singularity-free path. The second one is to, on-line, calculate the condition number of $\boldsymbol{D}$ and give a warning sign whenever it increases too fast, or halt the system when it reaches some pre-determined threshold. This way the platform is prevented from being operated in an ill-conditioned mode in which excessively large actuator forces are needed. In our experimental experience, we encounter no trouble solving (31). Thus the six forces $f_{P_{i_{x}}}, i=1,2, \ldots, 6$, are available to us.

Of course, as we explained in Section I, these forces are only approximations to the true interactions between the upper platform and the links. However, the analysis in (Wen et al., 1998a) reveals

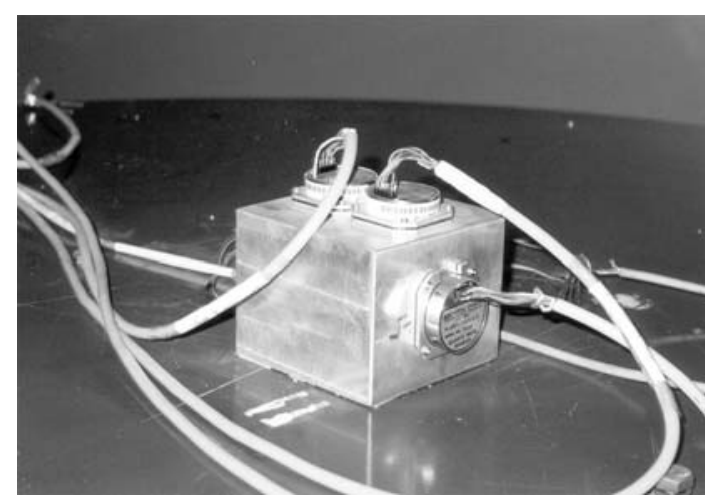

Fig. 3 Three angular rate gyroscopes on the upper platform

that the differences between dynamic and static forces are quite small for a typical motion. Furthermore, for our heavy hydraulic Stewart platform, operated at a moderate speed, the static forces are indeed the main interactions.

\section{ON-LINE ORIENTATION MEASUREMENT}

Our orientation measurement system comprises three solid-state rate gyroscopes manufactured by the Systron Donner Inertial Division of the BEI Sensors $\&$ Systems Company. Figure 3 is a picture of the hardware setup. It is seen, therein, that we employ the strapdown method and attach the sensors to the upper platform, with sensing directions of the three gyroscopes parallel to the three axes of the frame $\{P\}$, respectively. Since the three gyroscopes separately measure the roll, pitch, and yaw angular rates of the moving upper platform with respect to the base frame, the orientation information we need must be transformed from the measured signals. The outputs of the three gyroscopes are denoted as $P$ (roll rate), $Q$ (pitch rate), and $R$ (yaw rate). From the definitions of the frames introduced in Section II, if we define $\boldsymbol{\omega}_{P}=\dot{\alpha} \boldsymbol{k}_{B}+\dot{\beta} \boldsymbol{j}_{B^{\prime}}+\gamma \boldsymbol{i}_{B^{\prime \prime}}$, then we have (Lin, 1991)

$$
\begin{aligned}
\boldsymbol{\omega}_{P}= & {[\gamma-\alpha \sin (\beta)] \boldsymbol{i}_{B}+[\alpha \cos (\beta) \sin (\gamma)+\dot{\beta} \cos (\gamma)] \boldsymbol{j}_{B} } \\
& +[\alpha \cos (\beta) \cos (\gamma)-\dot{\beta} \sin (\gamma)] \boldsymbol{k}_{B} .
\end{aligned}
$$

Thus

$$
\boldsymbol{\omega}_{P}^{B}=\left[\begin{array}{c}
\gamma-\alpha \sin (\beta) \\
\alpha \cos (\beta) \sin (\gamma)+\dot{\beta} \cos (\gamma) \\
\alpha \cos (\beta) \cos (\gamma)-\dot{\beta} \sin (\gamma)
\end{array}\right]=\left[\begin{array}{c}
P \\
Q \\
R
\end{array}\right]
$$




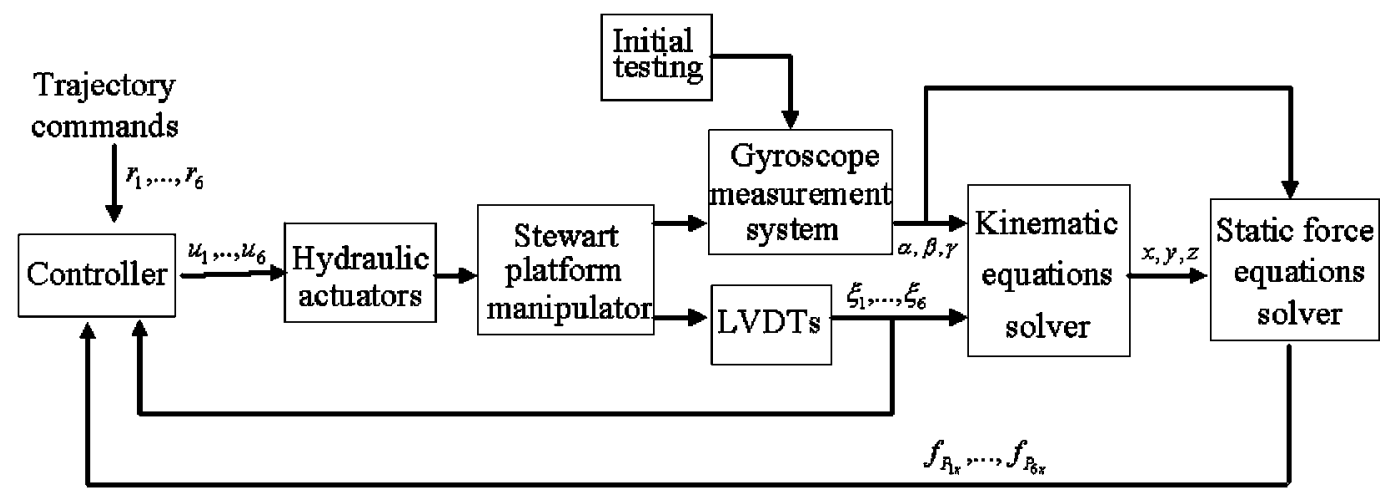

Fig. 4 Block diagram of the overall system

$$
\left[\begin{array}{c}
\alpha \\
\dot{\beta} \\
\gamma
\end{array}\right]=\left[\begin{array}{c}
\frac{1}{\cos (\beta)}[Q \sin (\gamma)+R \cos (\gamma)] \\
Q \cos (\gamma)-R \sin (\gamma) \\
P+\tan (\beta)[Q \sin (\gamma)+R \cos (\gamma)]
\end{array}\right]
$$

Note that for common Stewart platforms, including the one discussed in this paper, the angle $\beta$ is well limited inside the range of -90 degrees to 90 degrees. Therefore $\cos (\beta)$ is never zero and $\tan (\beta)$ is always finite in the above expression.

In our practical application, the continuous-time relationship (36) is discretized by the backward rectangular rule to the difference equations

$$
\left[\begin{array}{c}
\alpha_{n} \\
\beta_{n} \\
\gamma_{n}
\end{array}\right]=\left[\begin{array}{c}
\alpha_{n-1} \\
\beta_{n-1} \\
\gamma_{n-1}
\end{array}\right]+\left[\begin{array}{l}
\alpha^{\prime} \\
\beta^{\prime} \\
\gamma^{\prime}
\end{array}\right] \Delta t
$$

where $\alpha_{n}, \beta_{n}, \gamma_{n}$ are the values of $\alpha, \beta, \gamma$ at the $n$th sampling instant, $\alpha^{\prime}=\frac{1}{\cos \left(\beta_{n-1}\right)}\left[Q_{n} \sin \left(\gamma_{n-1}\right)+\right.$ $\left.R_{n} \cos \left(\gamma_{n-1}\right)\right], \beta^{\prime}=Q_{n} \cos \left(\gamma_{n-1}\right)-R_{n} \sin \left(\gamma_{n-1}\right), \quad \gamma=P_{n}+$ $\tan \left(\beta_{n-1}\right)\left[Q_{n} \sin \left(\gamma_{n-1}\right)+R_{n} \cos \left(\gamma_{n-1}\right)\right], P_{n}, Q_{n}, R_{n}$ are the values of $P, Q, R$ at the $n$th sampling instant, and $\Delta t$ is the sampling time. To generate the orientation information of the upper platform iteratively, this set of difference equations can be programmed on a computer in a straightforward manner, but to actually use it the bias problem of the rate gyroscopes must be taken care of first. Our experience shows that not only does the gyroscope have a bias in its outputs, but also the bias is time-varying, especially during the period the unit is warming up from a "cold start". Therefore, at the start of each experiment, we need to estimate the steady-state bias by averaging the outputs of the gyroscope over a period. The result is then deducted from $P_{n}, Q_{n}$, and $R_{n}$ subsequently. This procedure is called initial testing.

Figure 4 is a block diagram of the overall system.

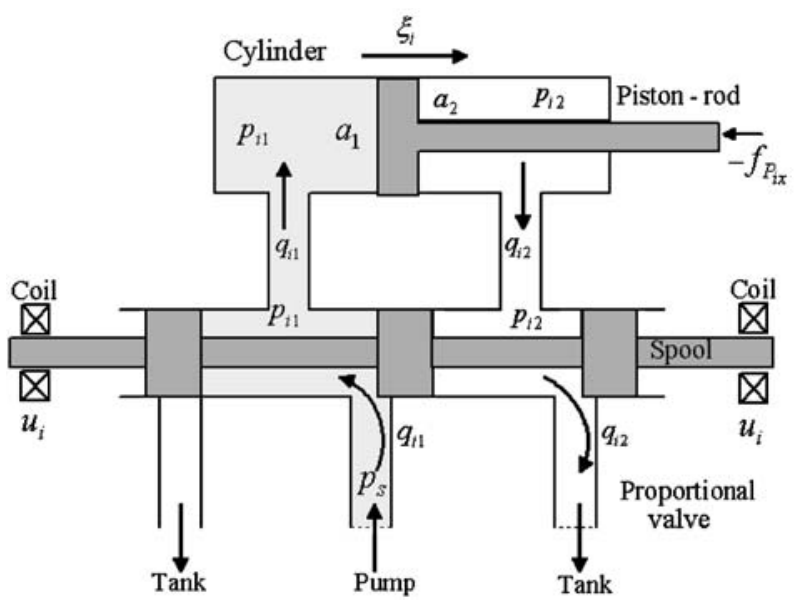

Fig. 5 The mechanical structure of the $i$ th cylinder and its associate proportional valve

Most blocks in the diagram have been explained by now, except the hydraulic actuator system and the controller, which are the subjects of the next Section.

\section{A SIMPLE HYDRAULIC ACTUATOR MODEL AND THE CONTROLLER DESIGN}

In the Stewart platform of Fig. 1, each link is a single-rod (or asymmetric) hydraulic cylinder actuated by a Bosch proportional valve. Since all six cylinder-valve systems are identical, only the mechanical structure of the $i$ th one is shown by the schematic diagram of Fig. 5, where a spool controlled by the electrical currents in the coils alters the oil flow direction and effective orifice sizes. The currents in the coils are the outputs of a compatible Bosch amplifier which responds linearly (within its operational range) to a voltage control signal. In the hydraulic system, a pump driven by a $10 \mathrm{HP}$ induction motor sends oil with a constant pressure $p_{s}$ to all six valves, and at the end of a cycle the oil flows out to a tank with a negligible pressure. 
In Fig. $5, q_{i 1}, q_{i 2}$, and $p_{i 1}, p_{i 2}$, are respectively oil flow rates and pressures at the designated areas. $a_{1}$ and $a_{2}$ are respectively the head and rod side areas of the piston. $\xi_{i}$ is the displacement of the $i$ th piston/ rod, which is equal to the link length $l_{i}$ minus the fixed length of the cylinder at its reset (shortest) position. $u_{i}$ is the control signal, i.e., the input voltage of the amplifier whose outputs are the currents in the coils. Finally $-f_{P_{i_{x}}}$ is the static force exerted by the upper platform on the $i$ th link, which is derived in Section II. Ignoring the leakage and compression effects of the working oil, as well as the inertial and frictional forces generated by the piston and rod, we can establish a dynamic model (Merritt, 1967; Wen et al., 1998b) for the cylinder-valve combination based on the orifice equations, flow continuity equations, and load equation:

$$
\begin{aligned}
& q_{i 1}=c_{w} \cdot \sqrt{p_{s}-p_{i 1}} \cdot u_{i}=a_{1} \dot{\xi}_{i}, \\
& q_{i 2}=c_{w} \cdot \sqrt{p_{2}} \cdot u_{i}=a_{2} \dot{\xi}_{i},
\end{aligned}
$$

for the "push" operation $\left(u_{i} \geq 0\right)$, or

$$
\begin{aligned}
& q_{i 1}=c_{w} \cdot \sqrt{p_{i 1}} \cdot u_{i}=a_{1} \dot{\xi}_{i}, \\
& q_{i 2}=c_{w} \cdot \sqrt{p_{s}-p_{i 2}} \cdot u_{i}=a_{2} \xi_{i},
\end{aligned}
$$

for the "pull" operation $(u \leq 0)$, where $c_{w}$ is the proportional constant of the actuator, and

$$
p_{i 1} a_{1}-p_{i 2} a_{2}=-f_{P_{i x}} .
$$

From the second equalities of (38) and (39) respectively, expressions for $p_{i 1}$ and $p_{i 2}$ can be formulated and substituted into (42) to form

$$
\xi_{i}=\frac{c_{w} \sqrt{p_{s} a_{1}+f_{P_{i x}}}}{\sqrt{a_{1}^{3}+a_{2}^{3}}} \cdot u_{i}
$$

for $u_{i} \geq 0$. Similarly, from (40), (41), and (42), it is straightforward to form

$$
\xi_{i}=\frac{c_{w} \sqrt{p_{s} a_{2}-f_{P_{i x}}}}{\sqrt{a_{1}^{3}+a_{2}^{3}}} \cdot u_{i}
$$

for $u_{i} \leq 0$.

The expressions (43) and (44) imply that the interaction between the upper platform and the $i$ th link is via the force $f_{P_{x_{x}}}$ only. Without it the length of each link could be controlled independently. Thus we are motivated to cancel the effect of the force term by adjusting the control signal $u$ accordingly.

Equipped with the information derived in the previous Sections, we propose to use the simple feedback control

$$
u_{i}(t)=k_{i}(t) \cdot\left[r_{i}(t)-\xi_{i}(t)\right]
$$

to cancel the effect of the force term, where $r_{i}(t)$ is a reference signal for the $i$ th link length change, and

$k_{i}(t)=\left\{\begin{array}{cc}\frac{\sqrt{a_{1}^{3}+a_{2}^{3}}}{\tau c_{w} \sqrt{p_{s} a_{1}+f_{P_{i_{x}}}(t)}} & r_{i}(t)-\xi_{i}(t) \geq 0, \\ \frac{\sqrt{a_{1}^{3}+a_{2}^{3}}}{\tau c_{w} \sqrt{p_{s} a_{2}-f_{P_{i_{x}}}(t)}} & r_{i}(t)-\xi_{i}(t)<0,\end{array}\right.$

is a time-varying gain. Then the closed-loop dynamic equation for the link becomes

$$
\dot{\xi}_{i}=-\frac{1}{\tau} \cdot \xi_{i}+\frac{1}{\tau} \cdot r_{i},
$$

which is a first-order system with the time constant $\tau$ and unity d.c. gain. Of course, in practical applications values of the system parameters like $c_{w}, p_{s}$, etc., are not known precisely, and there are measurement noises as well as computation errors that hinder the perfect estimation of $f_{P_{i_{x}}}$. Therefore, neither is it possible to make a link have the ideal linear time-invariant characteristic formulated in (47), nor it is possible to make all links have the identical, decoupled dynamic characteristic in practice. However, even the simplest constant gain feedback system has some robustness, so our goal is not to make an exact cancellation by $k(t)$, but only to make a reasonably accurate cancellation. The remaining question is how to choose the time constant $\tau$, which should not be too small due to the limited driving capability of the actuators. The question will be answered in the next Section.

\section{EXPERIMENT RESULTS}

Table 1 below summarizes physical parameter values of the Stewart platform and the hydraulic actuator system in the experiments. Though the system is essentially the same one in (Wen et al., 1998a, 1998b), the hydraulic actuator part has been slightly modified. Hence some parameter values are different in the present setup.

From Table 1 it is easy to see that $\xi_{i}=l_{i}-74.68$ when the unit is $\mathrm{cm}$. The six LVDTs used to measure $\xi_{i}, i=1,2, \ldots, 6$, convert a centimeter of length change to $0.2 \mathrm{~V}$ of output voltage. The three rate gyroscopes described in Section III have a measurement range of $-50 \% \mathrm{sec}$ to $+50 \% \mathrm{sec}$ that corresponds to the output range of $-2.5 \mathrm{~V}$ to $+2.5 \mathrm{~V}$. Signals from the LVDTs and gyroscopes are sent to a Pentium PRO 180 PC, 
Table 1 Various parameter values of the Stewart platform system

\begin{tabular}{|c|c|}
\hline Parameter & Value \\
\hline base radius $\left(R_{B}\right)$ : & $84 \mathrm{~cm}$ \\
\hline upper platform radius $\left(R_{P}\right)$ & $56 \mathrm{~cm}$ \\
\hline$\theta_{B}$ in Fig. 2(b): & $\frac{2}{9} \pi$ radian \\
\hline$\theta_{P}$ in Fig. 2(b): & $\frac{4}{9} \pi$ raian \\
\hline $\begin{array}{l}\text { weight of the upper } \\
\text { platform }\left(w_{P}\right) \text { : }\end{array}$ & $150 \mathrm{kgf}$ \\
\hline weight of each link $\left(w_{i}\right)$ : & $25 \mathrm{kgf}$ \\
\hline $\begin{array}{l}\text { length variation range } \\
\text { of each link }\left(l_{i}\right) \text { : }\end{array}$ & $74.68 \mathrm{~cm} \leq l_{i} \leq 124.68 \mathrm{~cm}$ \\
\hline piston head side area $\left(a_{1}\right)$ : & $19.635 \mathrm{~cm}^{2}$ \\
\hline piston rod side area $\left(a_{2}\right)$ : & $12.584 \mathrm{~cm}^{2}$ \\
\hline proportional constant $\left(c_{w}\right)$ : & $25 \mathrm{~cm}^{4} \mathrm{sec}^{-1}$ volt $^{-1} \mathrm{kgf}^{-1 / 2}$ \\
\hline supply pressure $\left(p_{s}\right)$ : & $12 \mathrm{kgf} / \mathrm{cm}^{2}$ \\
\hline
\end{tabular}

which performs all necessary computations to implement the control law with a sampling rate of $100 \mathrm{~Hz}$, and then sends out six control signals to the amplifiers of the proportional valves.

Before the control law (45) with the time-varying gain (46) is tested, a $150 \mathrm{kgf}$ payload is put on the upper platform at the center $O_{P}$. Then all links are commanded to lengthen equally, lifting the upper platform upwards with neither rotations nor horizontal translations. The control law adopted in this preparatory experiment is (45) with a constant gain $k_{i}(t)$ $=k$ for all links. Because the operation is symmetric in the sense that every link experiences the same force from the upper platform and the payload, tuning a single constant gain to use in all links is justifiable. From several trials the gain $k=k^{*}=1.8$ producing the most satisfactory response is recorded for later use.

In order to evaluate the performance of the proposed control scheme, we arrange two experiments. The first one tests only the basic upward motion, while the second one tests the other five degrees of freedom simultaneously. In both experiments, the control law (45) with a constant gain $k_{i}(t)=k^{*}=1.8$ for all links is applied first. Then the same law (45) with the time-varying gain (46) is applied for the $i$ th link, $i=1,2, \ldots, 6$. The results are compared. To make the distribution of forces on the links uneven, we change the payload into two graduate students, who weigh $150 \mathrm{kgf}$ in total approximately, and sit at the edge of the upper platform, on top of link 1. The picture in Fig. 6 depicts the arrangement, which affects the computation of static forces. For convenience, it is assumed that near the joint $P_{1}$, there is a point mass of $150 \mathrm{~kg}$. Thus the mass center of the upper platform and the payload can be determined accordingly and used to modify (28), as explained in Section II.

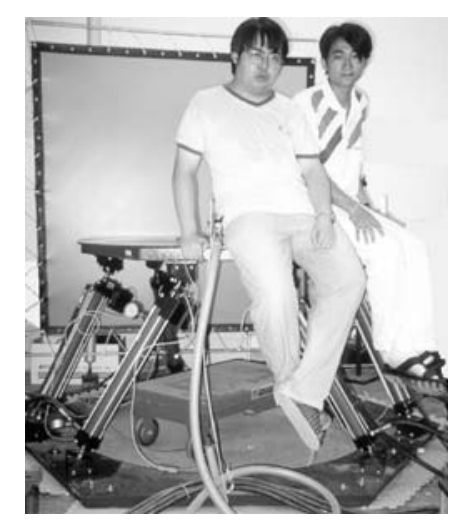

Fig. 6 Two graduate students act as the unbalanced payload

\section{Experiment 1.}

Let the motion trajectory command be

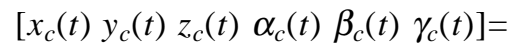

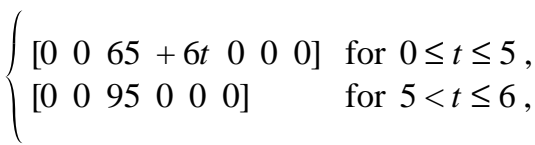

where units for $\left[x_{c}(t) y_{c}(t) z_{c}(t)\right]$ are cms, for $\left[\alpha_{c}(t)\right.$ $\left.\beta_{c}(t) \gamma_{c}(t)\right]$ are degrees, and for $t$ is seconds. This means that the upper platform and the unbalanced payload should be lifted from $65 \mathrm{~cm}$ to $95 \mathrm{~cm}$ at a speed of $6 \mathrm{~cm} / \mathrm{sec}$, without making any horizontal movements or rotations.

Up to now almost all formulas and data for calculating the time-varying gain (46) are available, except the value of $\tau$. As the ideal system characteristic (47) must be feasible and realistic, $\tau$ is chosen to satisfy

$$
k^{*}=\frac{\sqrt{a_{1}^{3}+a_{2}^{3}}}{\tau c_{w} \sqrt{p_{s} a_{1}+f_{P_{i_{x}}}^{a}}},
$$

where $f_{P_{i}}^{a}=57.4245 \mathrm{kgf}$ is the static force every link withstands when the upper platform carries a $150 \mathrm{kgf}$

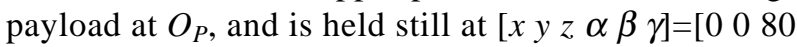
$\left.\mathrm{cm} \quad \begin{array}{lll}0 & 0 & 0\end{array}\right]$. Note that $80 \mathrm{~cm}$ is exactly the half way between $65 \mathrm{~cm}$ and $95 \mathrm{~cm}$. This choice of $\tau$ attempts to match the ideal characteristic to that of the system controlled by the constant gain in a representative operation situation. The resultant value of $\tau=0.1629$, and the time-varying gain for the $i$ th link is

$$
k_{i}(t)= \begin{cases}\frac{24.02}{\sqrt{235.62+f_{P_{i_{x}}}(t)}} & \text { for } r_{i}(t)-\xi_{i}(t) \geq 0, \\ \frac{24.02}{\sqrt{151.01-f_{P_{i_{x}}}(t)}} & \text { for } r_{i}(t)-\xi_{i}(t)<0,\end{cases}
$$




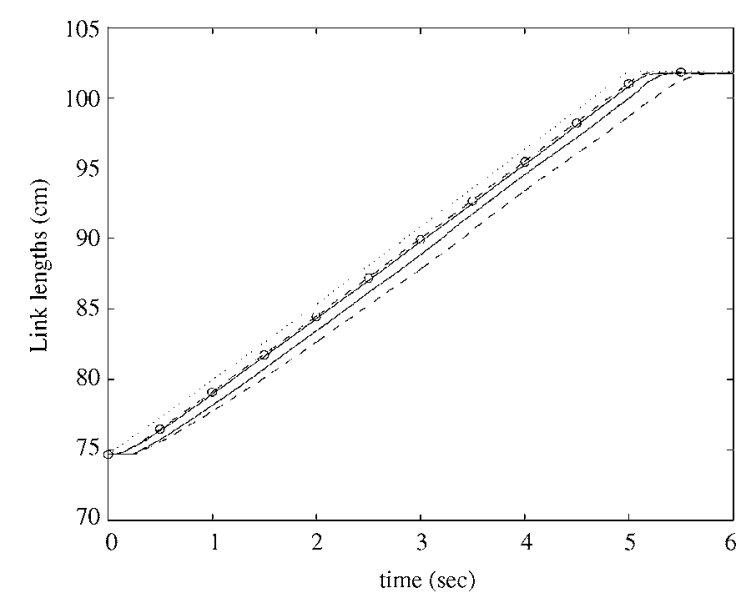

Fig. 7 Reference signal for the link length (dotted line), response of the ideal system (circles), fastest and slowest responses of the links controlled by the constant gain (dashed lines), and by the time-varying gain (solid lines)

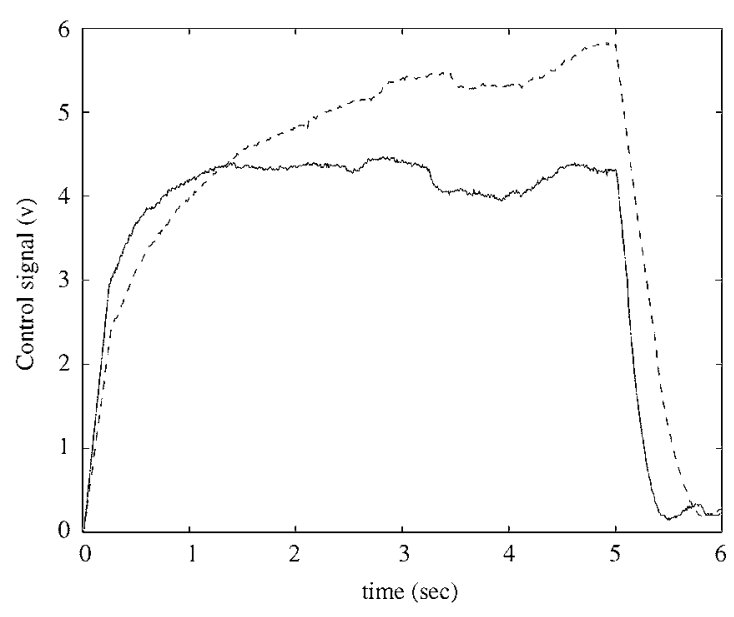

Fig. 8 Controls signals for link 6 generated by the constant gain controller (dashed line) and time-varying gain controller (solid line)

where $r_{i}(t)$ is the reference signal for the $i$ th link. In principle, for the above command (48) only "push" action is needed, unless there are overshoots, which are unlikely to happen. For the sake of safety, the gain formula for $r_{i}(t)-\xi_{i}(t)<0$ in $(50)$ is still programmed into the controller.

In response to the command (48) all links' lengths $l_{i}$ should increase uniformly from $74.68 \mathrm{~cm}$ (corresponding to $z(t)=65 \mathrm{~cm}$ ) to $101.86 \mathrm{~cm}$ (corresponding to $z(t)=95 \mathrm{~cm}$ ), but due to the unbalanced load, some links will respond faster than others. In Fig. 7, the dotted line is the reference signal $r_{i}(t)$ for all links to follow, the circles are the response of the ideal system (47) with $\tau=0.1629$, the solid lines
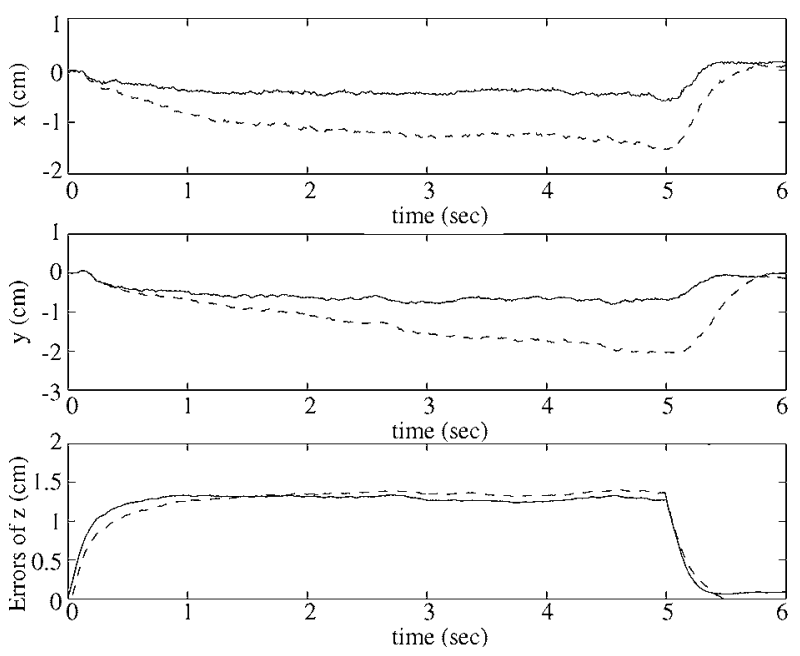

Fig. 9 Position trajectories $x(t), y(t)$ and tracking errors of $z(t)$ obtained from using the constant gain (dashed lines) and the time-varying gain (solid lines) respectively
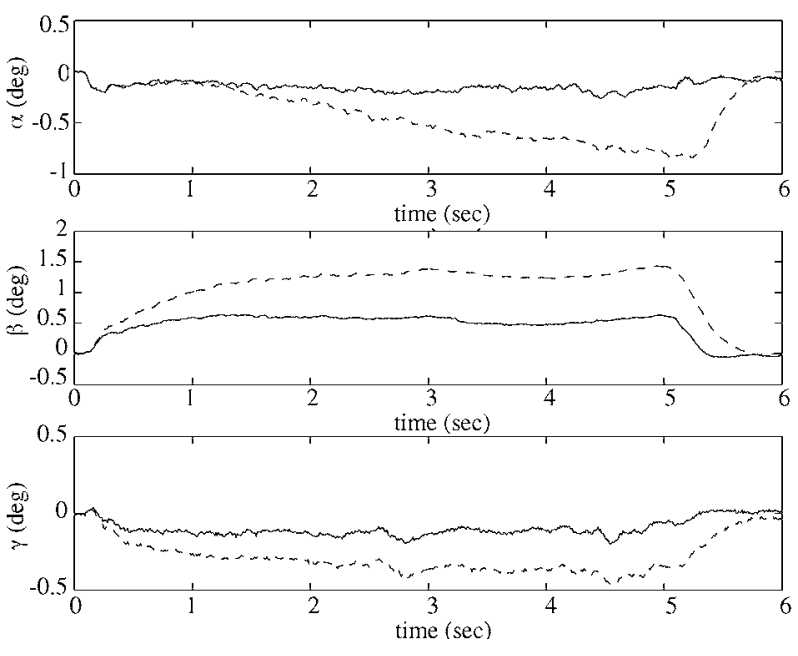

Fig. 10 Orientation trajectories $\alpha(t), \beta(t), \gamma(t)$ obtained from using the constant gain (dashed lines) and the time-varying gain (solid lines) respectively

are the measured responses of the fastest and slowest links controlled by the time-varying gain (50), and the dashed lines are the measured responses of the fastest and slowest links controlled by the constant gain $k^{*}=1.8$. First we see that all link responses are slower than that of the ideal system. This is attributed mainly to neglected frictional forces. Second, we see that the maximal difference between the fastest response and the slowest one with the constant gain is $2.393 \mathrm{~cm}$, while the difference with the timevarying gain is only $0.952 \mathrm{~cm}$. Obviously, letting each link adjust its own gain can cope with the unbalanced load better. This can also be seen in Fig. 8, where the control signals for link 6 are displayed. 

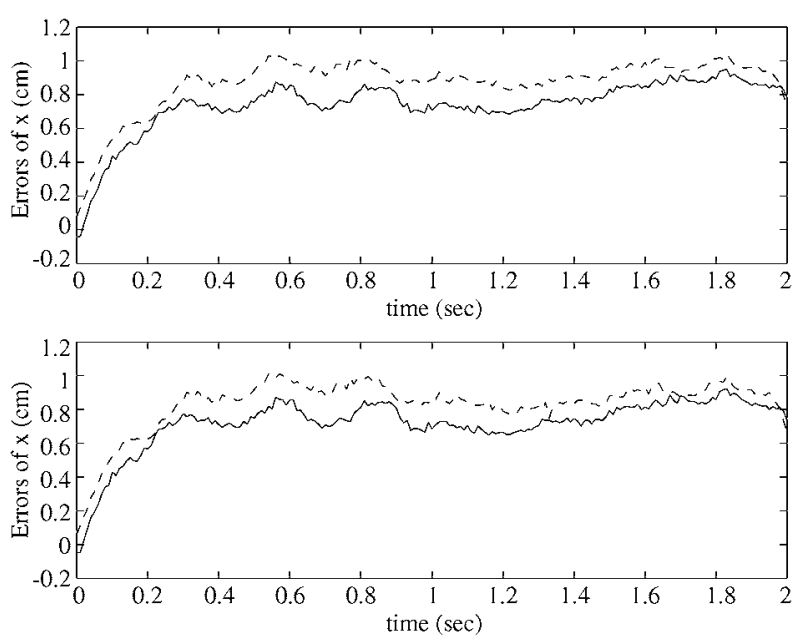

Fig. 11 Tracking errors of $x(t)$ obtained from using the constant gain (dashed lines) and the time-varying gain (solid lines) respectively

Contrary to our intuition, it is link 6 that withstands the largest force in our arrangement, not link 1 . This is due to the moment that link 6 has to produce. Fig. 8 tells us that the control signal from the constant gain controller is not large enough at first, and has to increase later on so that the enlarging tracking error can be reduced.

Figures 9 and 10 are respectively the position trajectories of $x(t), y(t)$, tracking error of $z(t)$, and the orientation trajectories of $\alpha(t), \beta(t), \gamma(t)$. These trajectories are obtained through off-line computations made after the experiment. The measured link lengths are inserted on the left sides of the kinematic Eq. (7), and the Newton-Raphson method is used to iteratively solve for the position and orientation variables. From the trajectories we see that ignoring unbalanced payload does not result in significant tracking error in the vertical displacement, but does cause the upper platform to move more, horizontally, and to rotate more.

It is worth mentioning that off-line analysis and computation reveal that the largest difference between static and dynamic forces along the trajectory in this experiment is within $10^{-5} \%$. This justifies the use of static forces approximation in the current experiment.

\section{Experiment 2.}

In this experiment, the upper platform carrying the unbalanced payload is brought to the state $\left[\begin{array}{lll}x & y & z\end{array}\right.$ $\alpha \beta \gamma]=\left[\begin{array}{lllllll}0 & 0 & 90 \mathrm{~cm} & 0 & 0 & 0\end{array}\right]$ first. Then the time is reset to $t=0$ and the motion trajectory command for the system to follow is

$$
\begin{aligned}
& {\left[\begin{array}{llllll}
x_{c}(t) & y_{c}(t) & z_{c}(t) & \alpha_{c}(t) & \beta_{c}(t) & \gamma_{c}(t)
\end{array}\right]} \\
& =\left[\begin{array}{lllllll}
5 t & 5 t & 90 & -2.5 t & -5 t & 5 t
\end{array}\right] \quad \forall 0 \leq t \leq 2,
\end{aligned}
$$
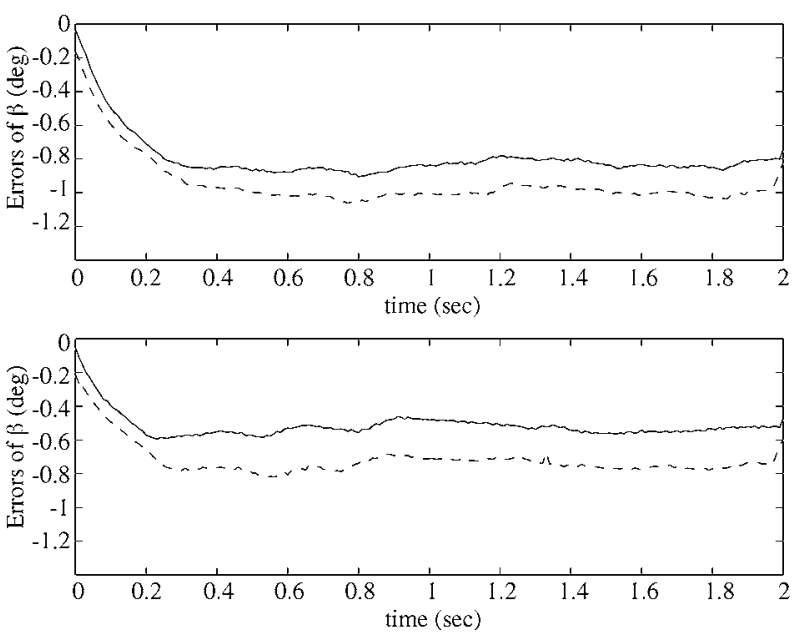

Fig. 12 Tracking errors of $\beta(t)$ obtained from using the constant gain (dashed lines) and the time-varying gain (solid lines) respectively

where the units are the same as those in (48). Clearly, this command is to test the responses of the system with respect to all degrees of freedom other than vertical displacement. Furthermore, it worsens the uneven force distribution among all links caused by the unbalanced payload, because it asks the upper platform to move outward, taking the payload farther from the original mass center of the entire system. For this command, the largest difference between static and dynamic forces along the trajectory is within $0.1 \%$, according to off-line simulation and analysis.

Here we re-select the ideal system time constant $\tau$ by requiring it to satisfy

$$
k^{*}=\frac{\sqrt{a_{1}^{3}+a_{2}^{3}}}{\tau c_{w} \sqrt{p_{s} a_{1}+f_{P_{i_{x}}}^{b}}},
$$

where $f_{P_{i}}^{b}=55.94 \mathrm{kgf}$ is the static force every link withstand's when the upper platform carries a $150 \mathrm{kgf}$ payload at $O_{P}$, and is held still at $\left[\begin{array}{llllll}x & y & z & \alpha & \beta & \gamma\end{array}\right]=\left[\begin{array}{ll}0 & 0\end{array}\right.$ $90 \mathrm{~cm} \quad 0 \quad 0 \quad 0]$, the initial state of the command (51). The resultant value of $\tau=0.1621$, and the time-varying gain for the $i$ th link is

$$
k_{i}(t)= \begin{cases}\frac{24.02}{\sqrt{235.62+f_{P_{i_{x}}}(t)}} & \text { for } r_{i}(t)-\xi_{i}(t) \geq 0, \\ \frac{24.02}{\sqrt{151.01-f_{P_{i_{x}}}(t)}} & \text { for } r_{i}(t)-\xi_{i}(t)<0,\end{cases}
$$

Similar to those of Experiment 1, the results of this experiment show that with the time-varying gain 
(53), the tracking errors are smaller than that with the constant gain $k^{*}=1.8$. For the sake of brevity, only Figs. 11 and 12 are given to show the tracking errors of the position variable $x(t)$ and those of the Euler angle $\beta(t)$, respectively.

In both Figs. 11 and 12 there are two diagrams each. The upper diagrams are obtained by using offline computations, like Figs. 9 and 10, while the lower diagrams are from data recorded during the experiments. More specifically, the lower diagram of Fig. 11 is from (14), and that of Fig. 12 is the outputs of the difference equations (37). Because only outputs of gyroscopes are used for on-line determination of the orientation variables, the mismatch between the upper diagram and the lower diagram in Fig. 12 is easier to detect. In contrast, for on-line determination of the translational variables, the link lengths are also used. Hence the lower diagram of Fig. 11 is closer to its off-line counterpart, which relies completely on the link lengths.

\section{CONCLUSIONS}

In this study, we explicitly formulate the interactions among different links of a Stewart platform through the forces acting on the links, and try to cancel, at least partially, these interactions by using a feedback control scheme which takes these interactions into consideration. To actually implement the scheme, we propose to use three gyroscopes to measure the orientations of the upper platform, and use the outputs along with link lengths to compute the static forces that the links must generate in the control loop. Our experiments show that the results are better than those obtained by using a single constant feedback gain for all links. In the future we shall investigate the effects of actuator saturation, which do not happen in the experiments described here because we limit the operation speed of the Stewart platform.

\section{ACKNOWLEDGEMENT}

This research is supported in part by the National Science Council of the R.O.C. under Grants NSC 862623-D-002-025 and NSC 89-2213-E-002-088.

\section{NOMENCLATURE}

$\begin{array}{ll}a_{1} & \text { piston head side area } \\ a_{2} & \text { piston rod side area } \\ c_{w} & \text { proportional constant } \\ f_{P_{i_{x}}} & \text { static force exerted by the upper platform } \\ k_{i}(t) \quad & \begin{array}{l}\text { on the } i \text { th link } \\ \text { controller gain for the } i \text { th link }\end{array} \\ l_{i} & \text { length of the } i \text { th link } \\ P, Q, R & \text { outputs of rate gyroscopes }\end{array}$

$p_{s} \quad$ supply pressure

$p_{i 1}, p_{i 2} \quad$ oil pressure of the $i$ th link

$q_{i 1}, q_{i 2} \quad$ oil flow rate of the $i$ th link

$R_{B} \quad$ base radius

$R_{P} \quad$ upper platform radius

$u_{i} \quad$ control signal for the $i$ th link

$w_{i} \quad$ weight of the $i$ th link

$w_{P} \quad$ weight of the upper platform

$x, y, z \quad$ position variables of the upper platform

$\{B\} \quad$ coordinate frame attached to the base

$\left\{J_{i}\right\} \quad$ coordinate frame attached to the $i$ th link

$\{P\} \quad$ coordinate frame attached to the upper platform

$\boldsymbol{i}_{P}, \boldsymbol{j}_{P}, \boldsymbol{k}_{P}$ three unit vectors of the frame $\{P\}$

$\boldsymbol{i}_{B}, \boldsymbol{j}_{B}, \boldsymbol{k}_{B}$ three unit vectors of the frame $\{B\}$

$\boldsymbol{i}_{i}, \boldsymbol{j}_{i}, \boldsymbol{k}_{i}$ three unit vectors of the frame $\left\{J_{i}\right\}$

$\boldsymbol{R}_{P}^{B} \quad$ the Euler orientation matrix

$\boldsymbol{R}_{B}^{J_{i}}, \boldsymbol{T}_{i}^{P} \quad$ coordinate transformation matrices

\section{Greek symbols}

$\alpha, \beta, \gamma \quad$ Euler angles of the upper platform

$\tau \quad$ time constant

$\xi_{i} \quad$ displacement of the $i$ th $\operatorname{rod}$

\section{REFERENCES}

1. Bhaskar, D., and Mruthyunjaya, T. S., 1998, "Singularity-Free Path Planning for the Stewart platform manipulator," Mechanism and Machine Theory, Vol. 33, No. 6, pp. 711-725.

2. Bhaskar, D., and Mruthyunjaya, T. S., 2000, "The Stewart Platform Manipulator: a Review," Mechanism and Machine Theory, Vol. 35, No. 1, pp. 15-40.

3. Charles, C. N., Antrazi, S. S., Zhou, Z.-L., and Campbell, C. E., 1993, "Adaptive Control of a Stewart Platform-Based Manipulator," Journal of Robotic Systems, Vol. 10, No. 5, pp. 657-687.

4. Cheok, K. C., Overholt, J. L., and Beck, R. R., 1993, "Exact Methods for Determining the Kinematics of a Stewart Platform Using Additional Displacement Sensors," Journal of Robotic Systems, Vol. 10, No. 5, pp. 689-707.

5. Do, W. Q. D., and Yang, D. C. H., 1988, "Inverse Dynamic Analysis and Simulation of a Platform Type of Robot," Journal of Robotic Systems, Vol. 5, No. 3, pp. 209-227.

6. Fichter, E. F., 1986, “A Stewart Platform-based Manipulator: General Theory and Practical Construction," International Journal of Robotic Research, Vol. 5, No. 2, pp. 157-182.

7. Grant, P. R., and Reid, L. D., 1997, "Motion Washout Filter Tuning: Rules and Requirements," Journal of Aircraft, Vol. 34, No. 2, pp. 145-151. 
8. Kosuge, K., Takeo, K., Fukuda, T., Kitayama, H., Takeuchi, N., and Murakami, H., 1996, "Force Control of Parallel Link Manipulator with Hydraulic Actuators," in Proceedings of the 1996 IEEE International Conference on Robotics and Automation, Minneapolis, MN, pp. 305-310.

9. Lin, C.-F., 1991, Modern Navigation, Guidance, and Control Processing, Englewood Cliffs, NJ: Prentice Hall, Inc.

10. Liu, K., Fewis, F. L., Lebret, G., and Taylor, D., 1993, "The Singularities and Dynamics of a Stewart Platform Manipulator," Journal of Intelligent Robotics and Systems, Vol. 8, No. 3, pp. 287-308.

11. Merritt, H. E., 1967, Hydraulic Control Systems, New York, NY: John Wiley \& Sons, Inc.

12. Salcudean, S. E., Drexel, P. A., Ben-Dov, D., Taylor, A. J., and Lawrence, P. D., 1994, "A Six Degree-of-Freedom, Hydraulic, One Person Motion Simulator," in Proceedings of the 1994 IEEE
International Conference on Robotics and Automation, San Diego, CA, pp. 2437-2443.

13. Tsai, L. W., 1999, Robot Analysis: the Mechanics of Serial and Parallel Manipulator, New York, NY: John Wiley \& Sons, Inc.

14. Wen, C., Hsu, C.-C., and Fong, I-K., 1998a, "Modeling and Control of a Hydraulic Stewart Platform Part I: Kinematics and Dynamics Analysis," Journal of Control System and Technology, Vol. 6, No. 3, pp. 177-184.

15. Wen, C., Hsu, C.-C., and Fong, I-K., 1998b, "Modeling and Control of a Hydraulic Stewart Platform Part II: Modeling and Control of the Hydraulic Links," Journal of Control System and Technology, Vol. 6, No. 3, pp. 185-192.

Manuscript Received: Nov. 20, 2000

Revision Received: Feb. 26, 2001 and Accepted: May 21, 2001

\title{
液壓史都華平台利用計算力回授之運動控制
}

\author{
許智欽 馮蟻剛
}

國立台灣大學電機工程學系

\section{摘 要}

在由六支油壓連桿驅動之史都華平台上, 我們架設三個陀螺儀感測器, 以 測得上平台運動之角速度。由此角速度訊號及線性電阻尺所測得之連桿長度訊 號, 我們可即時計算上平台質心之位置與姿態, 以及各連桿所承受之靜力大 小。這些資訊可加於各連桿之動態模式中, 藉以即時計算迴授增盆而控制連桿 長度。在一個具非平衡性負載之實驗中, 利用此法所得增益的實驗結果較使用 常數增六之結果爲優。

關鍵詞：史都華本台，回授控制，陀螺儀。 